\title{
Inflammatory cytokines in midbrain periaqueductal gray contribute to diabetic induced pain hypersensitivity through phosphoinositide 3-kinase/protein kinase B/mammalian target of rapamycin signaling pathway
}

\author{
Mochi Guo ${ }^{1, *}$, Zongming Jiang ${ }^{1, *}$, Yonghao Chen $^{2}$, Fei Wang ${ }^{3}$, and Zhifeng Wang ${ }^{4}$ \\ 'Department of Anesthesia, Shaoxing People's Hospital (Shaoxing Hospital, Zhejiang University School of Medicine), Shaoxing, Zhejiang, China \\ ${ }^{2}$ Department of Anesthesia, Kowloon Hospital, Shanghai Jiaotong University School of Medicine, Suzhou, Jiangsu, China \\ ${ }^{3}$ Bioinformation Branch, Hangzhou Hibio Bioinformation Technology Company Hang Zhou, Hangzhou, Zhejiang, China \\ ${ }^{4}$ Department of Anesthesia, Shaoxing Second Hospital, Shaoxing, Zhejiang, China
}

Received November 9, 2020

Revised December 22, 2020

Accepted December 24, 2020

Handling Editor: Jong Yeon Park

\section{Correspondence}

Zhifeng Wang

Department of Anesthesia, Shaoxing

Second Hospital, 123 Yanan Road,

Yuecheng District of Shaoxing City,

Shaoxing, Zhejiang 312000, China

Tel: +86-10-575-88229212

Fax: +86-10-575-88229286

E-mail: jixueyang1980@163.com

*These authors contributed equally to this work as co-first authors.
Background: Diabetes-related neuropathic pain frequently occurs, and the underpinning mechanism remains elusive. The periaqueductal gray (PAG) exhibits descending inhibitory effects on central pain transmission. The current work aimed to examine whether inflammatory cytokines regulate mechanical allodynia and thermal hyperalgesia induced by diabetes through the phosphoinositide 3-kinase (PI3K)-mammalian target of rapamycin (mTOR) pathway in the PAG.

Methods: Streptozotocin (STZ) was administered intraperitoneally to mimic allodynia and hyperalgesia evoked by diabetes in rats. Behavioral assays were carried out for determining mechanical pain and thermal hypersensitivity. Immunoblot and ELISA were performed to examine PAG protein amounts of interleukin-1 $\beta$ (IL-1 $\beta$ ), IL6 , and tumor necrosis factor- $\alpha(\mathrm{TNF}-\alpha)$, as well as their corresponding receptors in STZ rats, and the expression of PI3K/protein kinase B (Akt)/mTOR signaling effectors.

Results: Increased PAG p-PI3K/p-Akt/p-mTOR protein amounts were observed in STZ-induced animals, a PI3K-mTOR pathway inhibition in the PAG attenuated neuropathic pain responses. Moreover, the PAG concentrations of IL-1 $\beta, I L-6$, and TNF- $\alpha$ and their receptors (namely, IL-1R, IL-6R, and tumor necrosis factor receptor [TNFR] subtype TNFR1, respectively) were increased in the STZ rats. Additionally, inhibiting IL-1R, IL-6R, and TNFR1 ameliorated mechanical allodynia and thermal hyperalgesia in STZ rats, alongside the downregulation of PI3K-mTOR signaling.

Conclusions: Overall, the current study suggests that upregulated proinflammatory cytokines and their receptors in the PAG activate PI3K-mTOR signaling, thereby producing a de-inhibition effect on descending pathways in modulating pain transmission, and eventually contributing to neuropathic pain.

Key Words: Cytokines; Diabetes Mellitus; Hyperalgesia; Mesencephalon; Neuralgia; Pain Threshold; Periaqueductal Gray; Phosphatidylinositol 3-Kinases; Streptozocin; TOR Serine-Threonine Kinases. (a) This is an open-access article distributed under the terms of the Creative Commons Attribution Non-Commercial License (http://creativecommons.org/licenses/by-nc/4.0/), which permits unrestricted non-commercial use, distribution, and reproduction in any medium, provided the original work is properly cited.

(c) The Korean Pain Society, 2021
Author contributions: Mochi Guo: Methodology; Zongming Jiang: Investigation; Yonghao Chen: Writing/manuscript preparation; Fei Wang: Investigation; Zhifeng Wang: Funding acquisition. 


\section{INTRODUCTION}

Clinically, painful diabetic neuropathy frequently occurs in diabetic peripheral neuropathy cases $[1,2]$. Neuropathic pain is likely caused by peripheral or central nervous (CNS) system (i.e., brain and spinal cord) pathologies [3-5]. Measures to counteract and tackle abnormal sensations and pain remain scarce due to limited knowledge.

The mammalian target of rapamycin (mTOR) represents a ubiquitous protein, which contains two basic components, i.e., mTORC1 and mTORC2 [6]. It is admitted that mTOR and its related signaling networks are closely associated with nociceptive processes [7-12]. However, its role in the modulation of diabetic neuropathic pain in the CNS remains unclear. Furthermore, it is unknown whether CNS dysfunction contributes to the development of chronic pain and perception during diabetes. Additionally, as a downstream event of the phosphatidylinositol 3-kinase (PI3K)-mTOR signaling pathway [6], the regulation of pain sensitization by the PI3K-mTOR pathway through a central mechanism warrants further assessment.

Altered brainstem pain modulation circuits highly contribute to the generation of chronic pain and the related perceptions. The midbrain periaqueductal gray (PAG) constitutes an important part of the central descending pain modulation network, inhibiting or promoting pain transmission through the rostral ventromedial medulla that projects to the spinal dorsal horn [13-15]. Therefore, our primary goal was to investigate the potential mechanism by which PI3K-mTOR signaling alterations in the PAG affect diabetes-related mechanical and cold hypersensitivity. In this study, we hypothesized that PI3K-mTOR signaling is essential for regulating and generating diabetic neuropathic pain. Meanwhile, neuroinflammation and central sensitization are often implicated in neuropathic pain [16].

Diabetic pain is often elicited and maintained by peripherally or centrally driven inflammation. Neuropathic pain is commonly characterized by elevated amounts of inflammatory cytokines, especially the classical proinflammatory cytokines, namely tumor necrosis factor- $\alpha$ (TNF- $\alpha$ ), interleukin-1 $\beta$ (IL-1 $\beta$ ), and IL-6, through the activation of glia, including microglia and astrocytes [3]. The release of inflammatory cytokines by induced astrocytes and microglia causes neuronal cell exacerbation in pain modulation-associated brain regions [3]. These cytokines have been identified in related brain areas in human patients and animal models of diabetic mellitus $[17,18]$.

In this work, we speculated that inflammatory cytokines contribute to diabetic neuropathic pain, and that the concentrations of inflammatory cytokines in the PAG impact pain responses and intensities. We further hypothesized that PAG inflammatory cytokines and respective receptors are upregulated, and that cytokine signaling blockade in the PAG would improve responses to pain stimulation in diabetic rats via PI3K-mTOR signaling.

\section{MATERIALS AND METHODS}

\section{Animals}

Specific-pathogen-free male Sprague-Dawley rats (200$250 \mathrm{~g}$ ) were provided by Shanghai SLAC Laboratory Animal Center, Co. Ltd. (Shanghai, China). The animals were housed in climate-controlled cages at $20^{\circ} \mathrm{C}-25^{\circ} \mathrm{C}$ and $40 \%$ $70 \%$ humidity, under a $12 \mathrm{~h} / 12 \mathrm{~h}$ light-dark cycle, with food and water ad libitum. The animal protocols followed the guidelines of the International Association of the Study of Pain (in accordance with the UK Animal Act) and had approval from the Ethics Committee of Shaoxing People's Hospital (Shaoxing Hospital, Zhejiang University School of Medicine) (approval number: 2020-79). The rats underwent adaptive housing for 4 to 5 days before the start of experimental interventions.

\section{Diabetes model establishment}

A model of streptozotocin (STZ)-induced diabetes was established, based on a previous study [19]. In brief, 45-60 $\mathrm{mg} / \mathrm{kg}$ of STZ was injected intraperitoneally (i.p.) to induce diabetes. On an Auto Hematology Analyzer (MED-L-900; Youte Medical Instrument Company, Guangzhou, China), the levels of blood glucose were measured after an injection of STZ in blood specimens obtained from the tail vein, to assess the suitability of the model for the study. The model was considered to be suitable with blood glucose levels above $350 \mathrm{mg} / \mathrm{dL}$, after fasting for 3 hours, in rats within 5 weeks post-STZ administration, and used to further examine the effects of inflammatory cytokines in this study.

\section{Central cannulation and microinjection}

Rats underwent anesthesia by sodium pentobarbital injection $(45 \mathrm{mg} / \mathrm{kg}$, i.p.) and fixation in a stereotaxic apparatus for central cannulation and microinjection [20]. According to previously published reports, the following stereotaxic coordinates were used for the dorsolateral PAG (dl-PAG): 7.6 $\mathrm{mm}$ posterior to the bregma, $0.65 \mathrm{~mm}$ lateral to the midline, and $4.2 \mathrm{~mm}$ ventral to the brain surface. The guide cannula ( $0.8 \mathrm{~mm}$ outer diameter) was implanted and attached to the skull bone with stainless screws and cement (No. 700-00123-00, Screws-M 1.2 L 2.0 mm). A dummy 
cannula was inserted into and pulled out of the guide cannula twice a day for clogging prevention. After 3-5 days of recovery, drug infusion was initiated in the animals. After this period, cannula connection to an osmotic minipump (Model 2004/2006, Alzet Osmotic Pumps; DURECT Corporation, Cupertino, CA) was carried out to deliver the vehicle, artificial cerebrospinal fluid (aCSF), the mTOR antagonist (rapamycin; Sigma-Aldrich, St. Louis, MO) and a PI3K suppressor (LY294002; Sigma-Aldrich), respectively. Similarly, each inflammatory cytokine receptor antagonist (IL-1Ra, SC144; TNF- $\alpha$, etanercept, or ETAN) (Tocris Co., Ellisville, MO) was loaded into the pump. These drugs were diluted in aCSF to obtain the target concentration. Rapamycin and LY294002 were administered at a concentration of $300 \mu \mathrm{M}$, and proinflammatory cytokine receptor antagonists were utilized at a concentration of $10 \mu \mathrm{M}$ at $0.25 \mu \mathrm{L} /$ hour (Alzet Model 1003D/3 day-delivery; DURECT Corporation). The injection catheter was kept in place for at least five minutes for minimizing drug reflux during catheter removal. The rats were euthanized and decapitated for brain tissue dissection under a microscope after the completion of drug administration. Behavioral tests were carried out 1 hour prior to PAG infusion completion. Three tests were performed at 30-minutes intervals.

\section{Assessment of behavioral pain responses}

In accordance with methods provided by Um et al. [21], mechanical paw withdrawal threshold (PWT) was assessed with an electronic von Frey Anesthesiometer (IITC Company Life Science, Woodland Hills, CA) on rat hind paws. For the Von Frey test, individual rats were placed in a dedicated chamber on an elevated mesh floor with halves were painted in white and black, respectively. The apparatus was incorporated with rigid tips (testing pain threshold) with a $0.8 \mathrm{~mm}$ end-diameter filament to prevent any misreading. An unbending filament was applied to the rat hind paw vertically, with licking or quick withdrawal of the hindpaw considered a positive response. Six assays were performed within 3 minutes. In order to detect changes in thermal hyperalgesia, a radiant heat plate (IITC 39; IITC Company Life Science) was used for testing rat paw withdrawal latency (PWL). The rats were kept on the plates for 15 minutes for acclimatization, and to reduce their preference before each test. Three continuous stimulations were performed at 10-minutes intervals, and the average value reflected the PWL.

After the completion of all interventions, 2\% Evans blue $(0.25 \mu \mathrm{L})$ was injected via the cannula for site identification. Immediately, the rats were euthanized, and the midbrain was removed for dissection. Then, the specimens were immersed in a paraformaldehyde solution. The prop- er locations of the injection sites were confirmed by locating the Evans blue dye using histological analysis based on the atlas recommended by a previous study [21].

\section{Detection of PAG cytokines}

Tissues extracted from the PAG's dorsolateral regions under an anatomical microscope were homogenized in the RIPA buffer after all interventions. The homogenates were kept at $-80^{\circ} \mathrm{C}$. Commercially available enzyme-linked immunosorbent assay kits specific to TNF- $\alpha$ (Beijing Dingguo Changsheng Biotechnology Co., Ltd., Beijing, China), IL-6 (Jiankang Biological Engineering CO., Ltd., Shanghai, China), and IL-1 $\beta$ (Abcam, Cambridge, UK) were employed to assess cytokine levels in the supernatants as directed by the manufacturers.

\section{Tissue collection and immunoblot}

Rats were euthanized by i.p. administering sodium pentobarbital (100-120 mg/kg). The dl-PAG tissues were rapidly obtained from the brain and snap frozen in liquid nitrogen for homogenization. Sample processing was then performed as previously described [21]. Because biotin cannot penetrate through the cell membrane, it can exclusively biotinylate cell surface proteins. Unbound biotin was washed. PAG tissue samples then underwent homogenization and centrifugation $\left(12,000 \times \mathrm{g}, 12 \mathrm{~min} ; 4^{\circ} \mathrm{C}\right)$. Protein quantitation in supernatants utilized the bicinchoninic acid method. Protein samples $(200 \mu \mathrm{g})$ underwent incubation with streptavidin beads $(20 \mu \mathrm{L})$ for 3 hours at $4^{\circ} \mathrm{C}$, followed by washing with the RIPA buffer and centrifugation. The collected beads were boiled in sample buffer $(50 \mu \mathrm{L})$ for 3 minutes and centrifuged as described above. Equal amounts of total and membrane protein underwent sodium dodecyl sulfate polyacrylamide gel electrophoresis separation and electro-transfer onto polyvinylidene fluoride membranes. After blocking with $5 \%$ skim milk in tris buffered saline with Tween 20 for 1 hour at ambient, the membranes underwent successive incubations with rabbit primary antibodies against IL-1R, IL-6R, and TNFR1 (1:500; Neuromics, Edina, MN or Abcam), and horseradish peroxidase-conjugated anti-rabbit immunoglobulin G (1:250). Visualization was performed by enhanced chemiluminescence with the LAS 4000 reagent (GE Healthcare, Chicago, IL). Autoradiography was employed for detection and the NIH Scion image software (NIH, Bethesda, MA) was utilized for quantifying immunoreactive bands by densitometry. In a similar fashion, p-PI3K, p-Akt, and p-mTOR amounts as well as the levels of their non-phosphorylated forms were determined by immunoblot, with primary antibodies from Neuromics or Abcam. 


\section{Statistical analyses}

GraphPad Prism 8 (GraphPad Software, San Diego, CA) was employed for data analysis. Data are presented as mean standard error. One- or two-way analysis of variance and post-hoc Bonferroni, Tukey's, or Dunnett's tests were performed for comparisons. $P<0.05$ indicated statistical significance.

\section{RESULTS}

\section{Characterization of the diabetes model}

As shown in Fig. 1, a state of stable hyperglycemia was reached 3 weeks after intraperitoneal injection of STZ. This tended to be maintained thereafter, indicating that a successful diabetes model was established. As the blood glucose level increased, the mechanical threshold and latency of pain responses were amplified. STZ evidently decreased PWT and PWL compared with control values in rats 3-5 weeks post-treatment. Therefore, in this study, rats at 5 weeks post-STZ administration were selected as candidates to assess the impacts of inflammatory cytokine
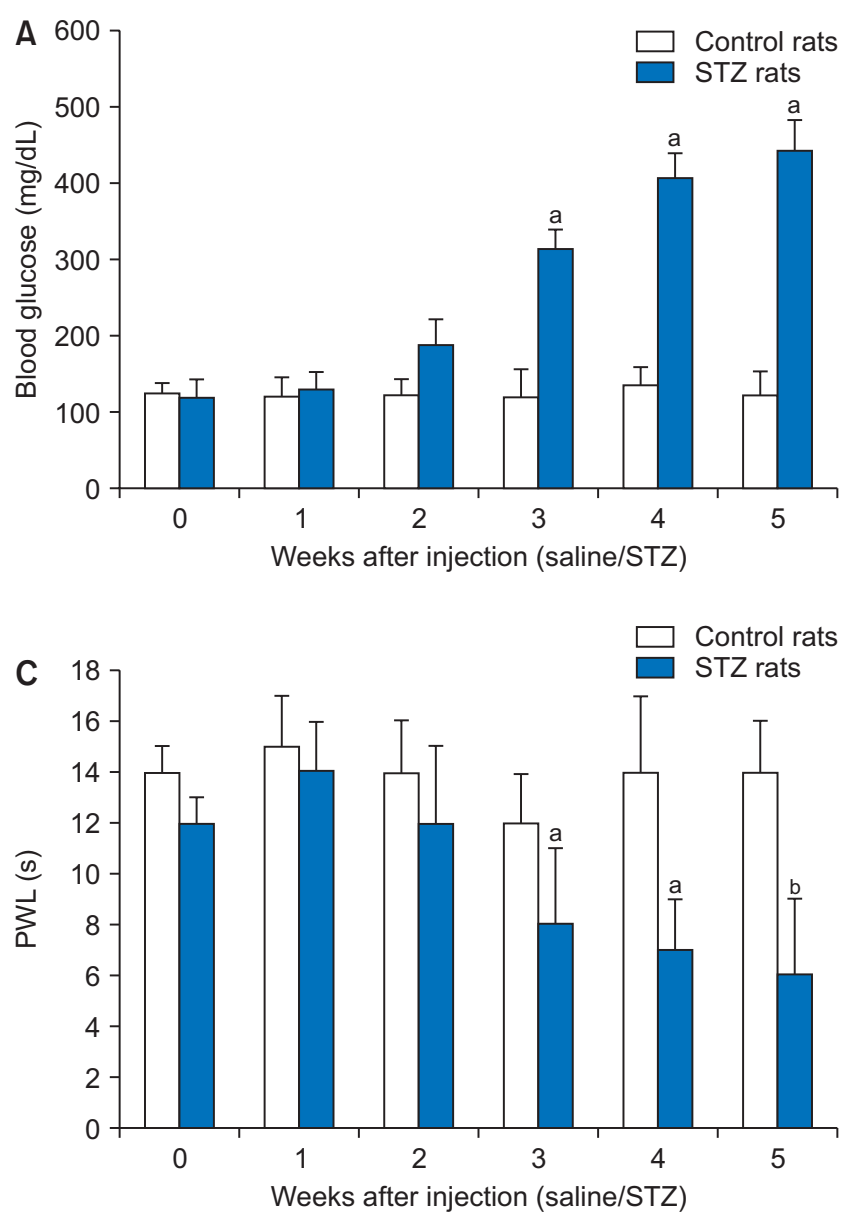

signaling.

\section{Involvement of the PI3K/Akt/mTOR pathway in the pain process}

As depicted in Fig. 2A, B, and C, remarkably increased amounts of phosphorylated p-PI3K, p-Akt, and p-mTOR were recorded after STZ administration in comparison with the control group $(n=8-10)$. Furthermore, PWT and PWL were significantly decreased in STZ-treated animals $(\mathrm{n}=12)$ in comparison with controls $(\mathrm{n}=12)$. However, no marked differences in total PI3K, Akt, or mTOR protein amounts were observed between the STZ and control groups. Rapamycin $(\mathrm{n}=10)$ and LY294002 $(\mathrm{n}=12)$ were used to block mTOR and PI3K, respectively, in the PAG. In this study, both PWT and PWL were markedly elevated during the 30-minutes test interval (Fig. 2D, E). Importantly, PWT and PWL were elevated in control animals compared with STZ rats receiving the above blockers.

\section{Roles of inflammatory cytokines in pain development}

Compared with control values, PAG concentrations of

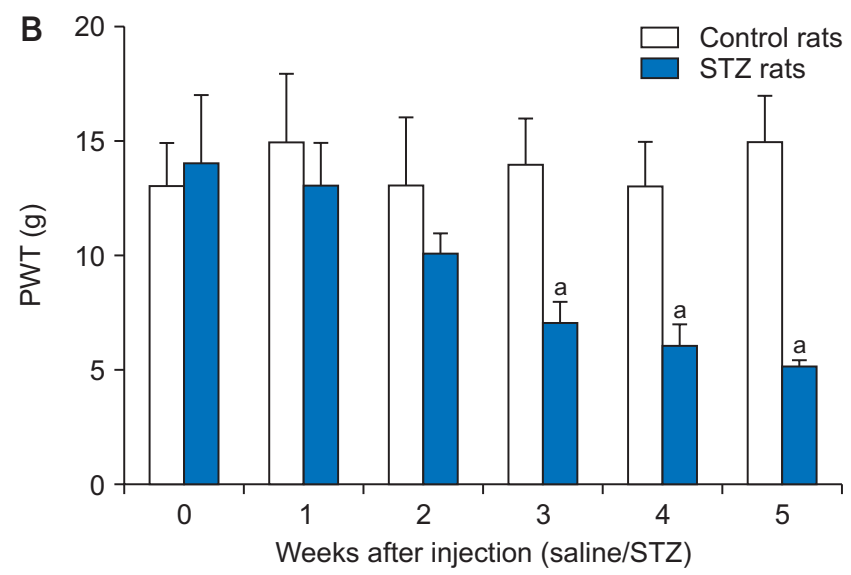

Fig. 1. Assessment of the streptozotocin (STZ)-induced diabetes model. (A) Hyperglycemia was developed in rats 3-5 weeks after STZ administration. (B, C) Both paw withdrawal threshold (PWT) and paw withdrawal latency (PWL) were significantly decreased 3 to 5 weeks following STZ injection. The error bars indicate standard deviation. ${ }^{\mathrm{a}} P<0.05$ and ${ }^{\mathrm{b}} P<$ 0.01 vs. control rats. 

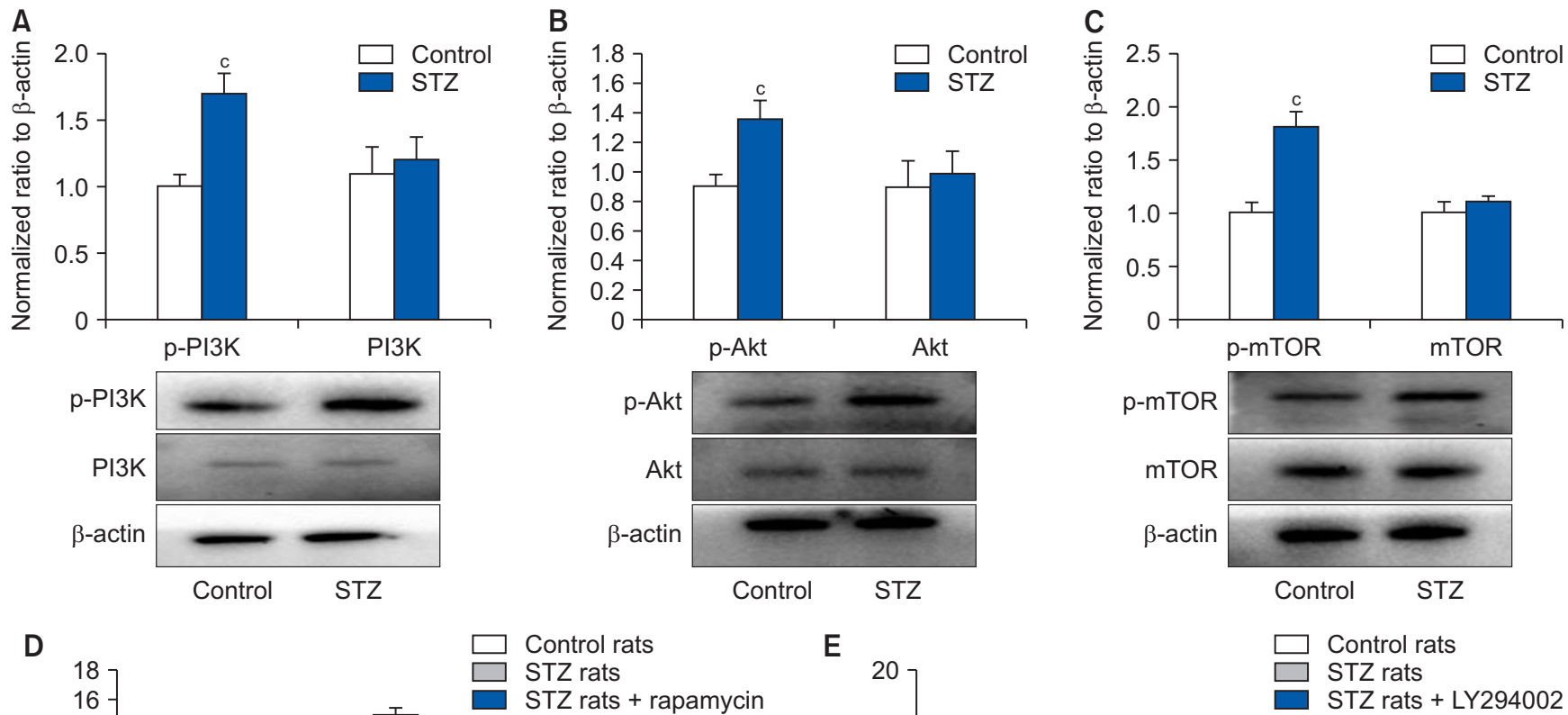

Control rats

STZ rats
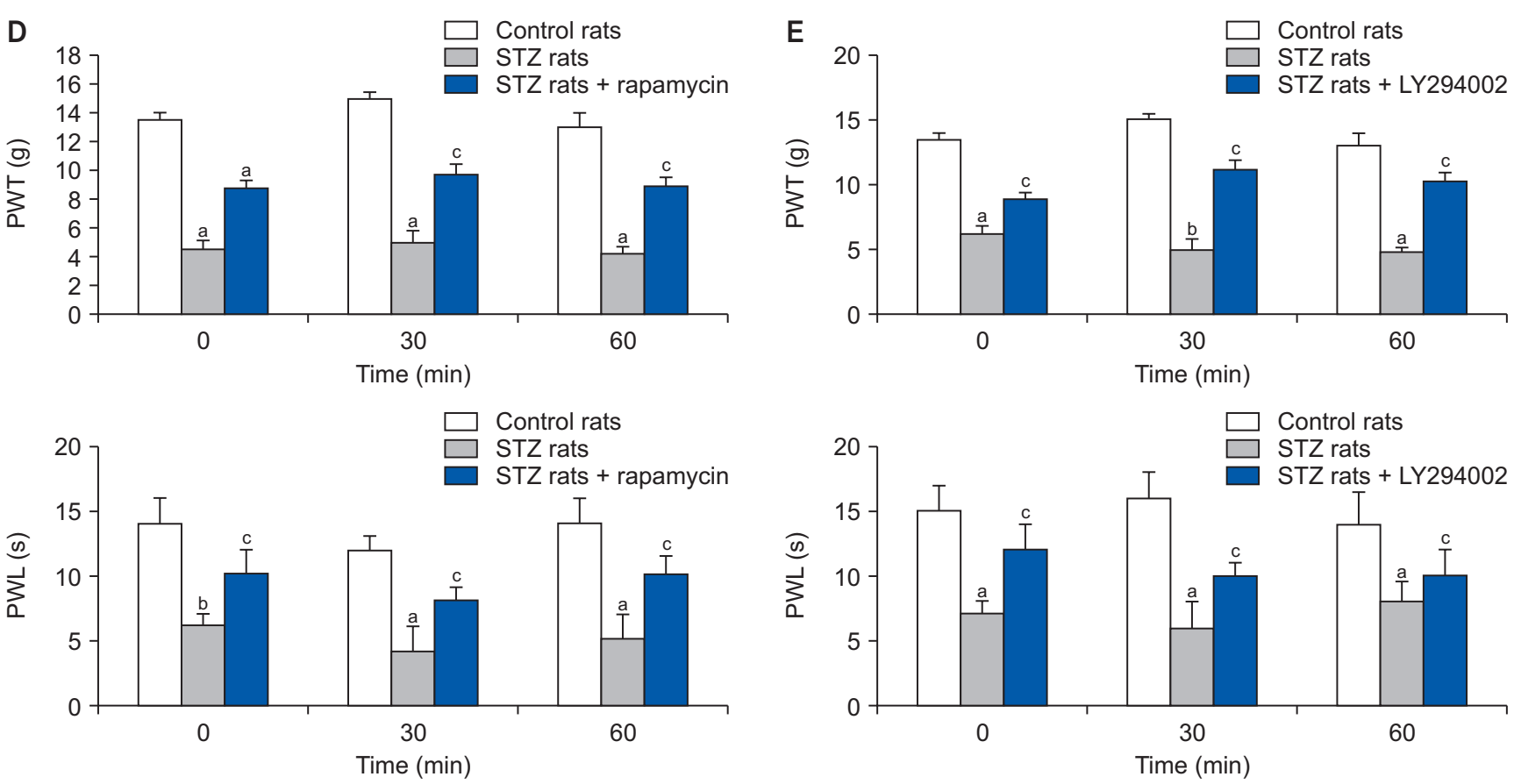

Fig. 2. Expression levels of phosphoinositide 3-kinase (PI3K)/protein kinase $B(A k t) / m a m m a l i a n$ target of rapamycin (mTOR) signaling effectors and pain responses after using blockers. (A-C) Phosphorylated PI3K ( $p$-PI3K), phosphorylated Akt ( $p$-Akt), and phosphorylated mTOR (p-mTOR) protein levels were increased in the periaqueductal gray (PAG) of streptozotocin (STZ) rats, whereas no statistical significance was observed in total PI3K, Akt, and mTOR protein amounts between the two groups. Values were normalized to $\beta$-actin. (D, E) Pain responses to mechanical and thermal stimulations after administration of LY294002 $(n=8)$ and rapamycin $(n=10)$ for blocking PI3K and mTOR, respectively in the PAG. The error bars indicate standard deviation. ${ }^{2} P$ $<0.05$ and ${ }^{\mathrm{b}} P<0.01$ vs. control rats. ${ }^{\mathrm{C}} P<0.05$ vs. STZ rats.

TNF- $\alpha$, IL-1 $\beta$, and IL- 6 were prominently elevated in STZ rats (Fig. 3A, $n=8$ ). Moreover, the expression levels of the respective cytokine receptors, namely TNFR1, IL-1R, and IL-6R, were also evidently upregulated in the membrane of the PAG tissue (Fig. 3B, C, n = 8-10). However, total PAG protein amounts of TNFR1, IL-1R, and IL-6R were similar in the control and STZ groups. The optical densities of TNFR1, IL-1R, and IL-6R protein bands were $1.08 \pm 0.21$, $0.98 \pm 0.20$, and $1.02 \pm 0.17$, respectively, in control animals, versus $1.01 \pm 0.15,1.11 \pm 0.12$, and $0.98 \pm 0.15$, respectively, in the STZ group ( $\mathrm{n}=8-10$ in each group).

In order to further determine the roles of cytokines in pain development and modulation, cytokine receptor blockers (ETAN, IL-1Ra, and SC144) were used to inhibit cytokine receptors in the PAG. The results showed that PWT and PWL in each corresponding antagonist group were remarkably increased during the three tests performed at 30-minutes intervals (Fig. 3D to F, STZ animals administered a given antagonist vs. non-treated STZ animals, $\mathrm{n}=8-10$ ). 

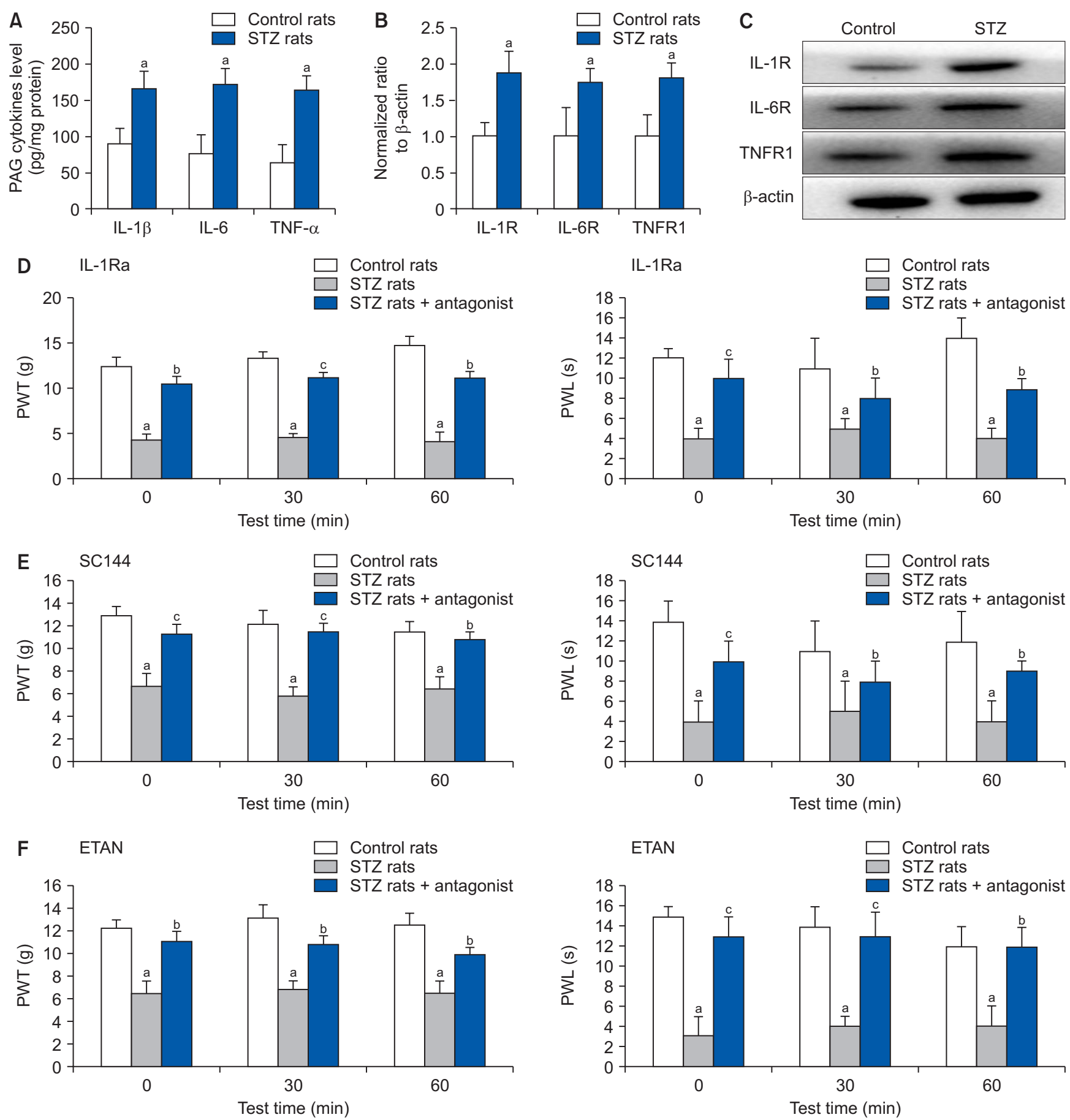

Fig. 3. Levels of cytokines and expression patterns of the corresponding receptors in the periaqueductal gray (PAG), and pain responses after receptor blocking. (A-C) Classical proinflammatory cytokines levels, the expression patterns of their receptors, and typical bands. There were increased levels of inflammatory cytokines and their receptors (interleukin [IL]-1R, IL-6R, and tumor necrosis factor receptor 1 [TNFR1]) in streptozotocin (STZ) rats ( $n=12)$. (D-F) After blocking their receptors, paw withdrawal threshold (PWT) and paw withdrawal latency (PWL) appeared to be reduced in STZ rats $(n=10)$ compared with control animals $(n=12)$. The error bars indicate standard deviation. ${ }^{a} P<0.01$ vs. control rats. ${ }^{b} P<0.05$ and ${ }^{c} P<0.01$ vs. STZ rats.

\section{PI3K and mTOR amounts after cytokine receptor blockade}

As shown in Fig. 4, injection of ETAN (TNF- $\alpha$ blocker) and SC144 (IL-1Ra blocker) into the PAG led to reduced p-PI3K and p-mTOR amounts in STZ-treated animals $(P<0.05$ vs. STZ animals without receptor inhibitor administration; $\mathrm{n}=6$-10/group). Interestingly, p-PI3K and p-mTOR amounts were similar in STZ rats receiving inhibitors and control animals. 

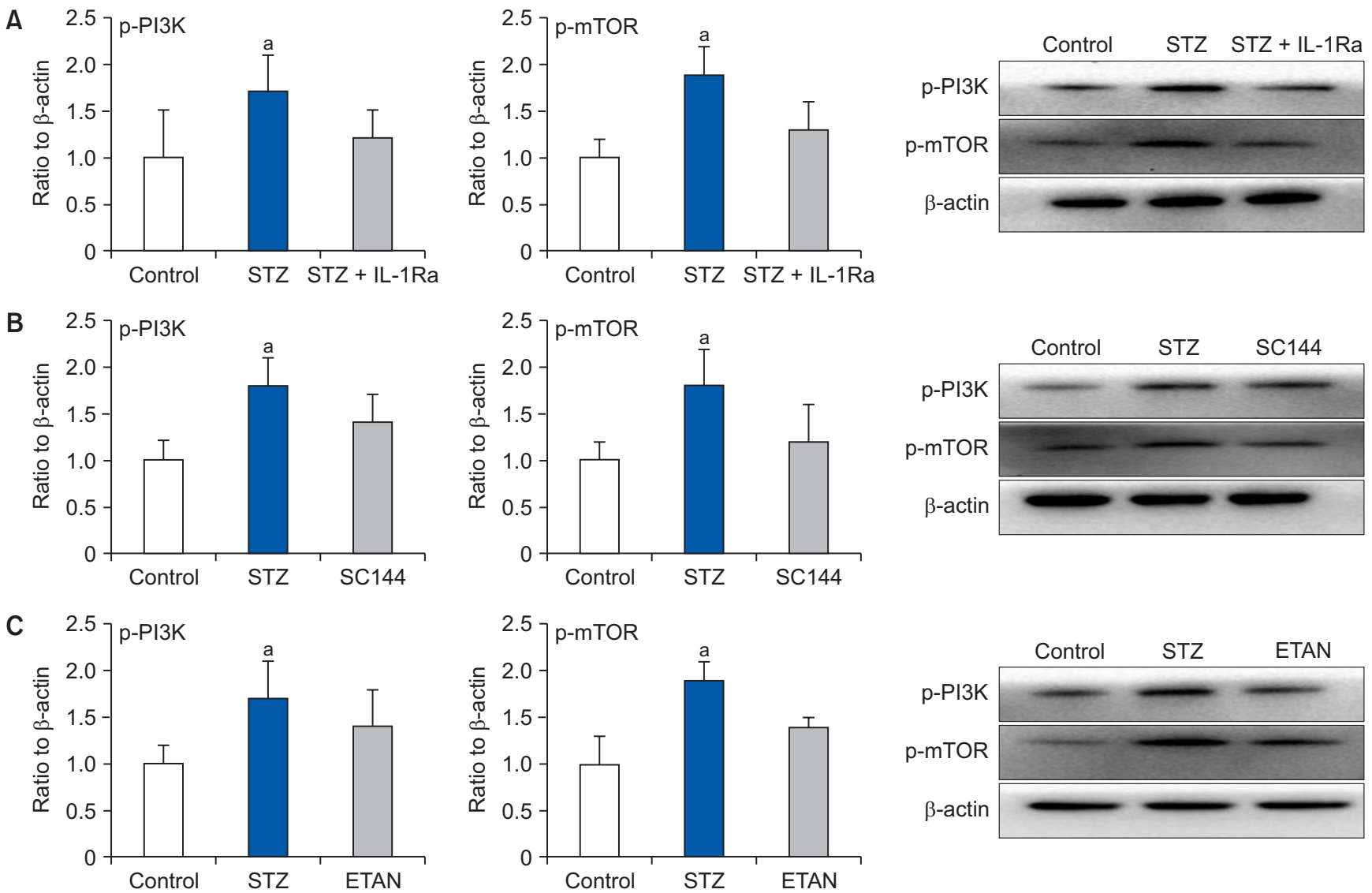

Fig. 4. Effects of cytokine receptor blockade on the expression of phosphorylated phosphoinositide 3-kinase (p-PI3K) and phosphorylated mammalian target of rapamycin (p-mTOR). (A-C) Infusion of the respective receptor inhibitors (interleukin [IL]-1Ra, SC144, and etanercept [ETAN]) into the periaqueductal gray resulted in decreased levels of p-PI3K and p-mTOR in streptozotocin (STZ) rats $(n=8)$. Interestingly, no differences were found between control animals and STZ rats administered these inhibitors $(n=12)$. The error bars indicate standard error. ${ }^{a} P<0.05$ vs. control rats $(n=8)$.

\section{DISCUSSION}

This study yielded the following results. 1) STZ increased the phosphorylation levels of PI3K, Akt, and mTOR in the PAG tissue. 2) STZ led to elevated membrane TNF- $\alpha$, IL$1 \beta$, and IL- 6 amounts in the PAG tissue. Notably, their corresponding receptors (TNFR1, IL-1R, and IL-6R) in the PAG tissue were also increased. 3) Blocking the signaling pathways in the PAG alleviated PWT and PWL reductions in STZ rats. 4) Blocking the proinflammatory receptors located in the PAG with ETAN and SC144 also attenuated pain responses to mechanical allodynia and thermal hyperalgesia in STZ rats; the possible mechanism might be linked to PI3K/Akt/mTOR pathway suppression.

It is widely believed that chronic pain is maintained in part by central sensitization, and promoted by neuroinflammatory reactions, which are characterized by glial cell activation, resulting in the secretion of proinflammatory cytokines and chemokines [3]. Diabetes often elicits terminal neuropathy and causes local inflammation, which eventually leads to chronic pain and even aberrant sensation $[22,23]$. In the CNS, the midbrain PAG is considered an essential part of the descending pain modulatory network, and inhibits or induces pain transmission and modulation [24]. Accumulating evidence reveals that morphine and kainic acid micro-injected into the PAG exert prolonged antinociceptive effects, and further studies have demonstrated that neuronal activity is partly regulated by signal input from the PAG [25]. Guo et al. [26] found that the neural substrates responsible for pain perception are located in the PAG of rats with neuropathic pain, pointing to the involvement of the midbrain PAG in pain responses. Moreover, a previous study revealed that the proinflammatory cytokines TNF- $\alpha$, IL-1 $\beta$, and IL- 6 contribute to pain modulation, with the upregulated proinflammatory cytokines impairing the descending inhibitory characteristics via the central GABAergic pathway in a Parkinson's disease rat model [27]. As demonstrated above, pain responses to mechanical and thermal stimuli were regulated by these proinflammatory cytokines via central PI3K/Akt/mTOR signaling.

Being central to host defense and inflammatory response, TNF functions depend on its two basic receptor subunits, i.e., TNFR1 and TNFR2 [28]. Unlike TNFR2, 
TNFR1 is exceptionally distributed in neuronal cells of the nervous system and exhibits normal function. Therefore, in this study, it obviously downregulated PAG PI3KmTOR in STZ rats and alleviated pain responses to stimuli, as shown by treatment with the specific TNFR1 inhibitor ETAN.

Traditionally, IL-6 is promptly and transiently produced in response to insults such as infections and tissue injury. Due to the membrane-bound property of IL-6 complexes, the majority of cells lack membrane-bound IL-6R, and consequently do not respond to IL- $6[29,30]$. Such cells react to IL-6 complexes using soluble IL-6R for activating glycoprotein 130 (a component of IL-6 signal transducers). Therefore, in this study, a glycoprotein 130 inhibitor called SC144 was injected into the PAG to suppress IL-6 related signal transduction with the goal of discerning whether IL-6 participates in PI3K/Akt/mTOR signaling and changes the responses to noxious stimuli. The final results showed that IL-6R amounts were elevated by STZ, and PI3K/Akt/ mTOR expression was downregulated after SC144 administration to the midbrain PAG. Moreover, PWT and PWL were attenuated by STZ administration.

It is widely accepted that IL-1 $\beta$ represents an important proinflammatory cytokine that is extensively involved in immune responses and signal transduction, both peripherally and centrally [31]. Corroborating previous discoveries [32], this study showed that STZ increased membrane IL-1R expression in the PAG, and blocking IL-1R significantly ameliorated pain responses, in both mechanical and thermal forms, while attenuating PI3K/Akt/mTOR signal transduction in the midbrain PAG of STZ treated rats. Furthering previous work [33,34], this study demonstrated that infusion of proinflammatory cytokine antagonists reduced the amplified pain responses in STZ animals, downregulating PI3K-mTOR signaling in the PAG. These findings provide a potential mechanism for the effects of cytokines in pain.

In conclusion, proinflammatory cytokines participate in the generation of neuropathic pain in STZ-treated (diabetic) rats. The potential underlying mechanism may be linked to alterations in the descending pain inhibitory effect through PI3K-mTOR signaling in the PAG, thereby highlighting a promising method for counteracting the protracted pain in patients with diabetes mellitus.

\section{ACKNOWLEDGEMENTS}

The authors thank Prof. Zhang Ling, a senior statistician at Shaoxing People's Hospital, for her critical review and explaining the data.

\section{CONFLICT OF INTEREST}

No potential conflict of interest relevant to this article was reported.

\section{FUNDING}

This research was supported by Zhejiang Provinicial Natural Science Foundation of China under Grant No: LY19H090006.

\section{ORCID}

Mochi Guo, https://orcid.org/0000-0003-4024-9452

Zongming Jiang, https://orcid.org/0000-0002-7187-0842

Yonghao Chen, https://orcid.org/0000-0001-7496-4492

Fei Wang, https://orcid.org/0000-0003-4066-287X

Zhifeng Wang, https://orcid.org/0000-0001-8873-8613

\section{REFERENCES}

1. Vinik AI. Clinical practice. Diabetic sensory and motor neuropathy. N Engl J Med 2016; 374: 1455-64.

2. Armstrong DG, Boulton AJM, Bus SA. Diabetic foot ulcers and their recurrence. N Engl J Med 2017; 376: 2367-75.

3. Ji RR, Nackley A, Huh Y, Terrando N, Maixner W. Neuroinflammation and central sensitization in chronic and widespread pain. Anesthesiology 2018; 129: 343-66.

4. Morgado C, Terra PP, Tavares I. Neuronal hyperactivity at the spinal cord and periaqueductal grey during painful diabetic neuropathy: effects of gabapentin. Eur J Pain 2010; 14: 693-9.

5. Morgado C, Silva L, Pereira-Terra P, Tavares I. Changes in serotoninergic and noradrenergic descending pain pathways during painful diabetic neuropathy: the preventive action of IGF1. Neurobiol Dis 2011; 43: 275-84.

6. Lisi L, Aceto P, Navarra P, Dello Russo C. mTOR kinase: a possible pharmacological target in the management of chronic pain. Biomed Res Int 2015; 2015: 394257.

7. Santini E, Huynh TN, Klann E. Mechanisms of translation control underlying long-lasting synaptic plasticity and the consolidation of long-term memory. Prog Mol Biol Transl Sci 2014; 122: 131-67.

8. Guo JR, Wang H, Jin XJ, Jia DL, Zhou X, Tao Q. Effect and mechanism of inhibition of PI3K/Akt/mTOR signal pathway on chronic neuropathic pain and spinal microglia in a rat model of chronic constriction injury. Oncotarget 2017; 8: 52923-34.

9. Price TJ, Rashid MH, Millecamps M, Sanoja R, Entrena JM, Cervero F. Decreased nociceptive sensitization in mice lacking the fragile $\mathrm{X}$ mental retardation protein: role of mGluR1/5 
and mTOR. J Neurosci 2007; 27: 13958-67.

10. Xu Q, Fitzsimmons B, Steinauer J, O'Neill A, Newton AC, Hua $\mathrm{XY}$, et al. Spinal phosphinositide 3-kinase-Akt-mammalian target of rapamycin signaling cascades in inflammationinduced hyperalgesia. J Neurosci 2011; 31: 2113-24.

11. Asante CO, Wallace VC, Dickenson AH. Formalin-induced behavioural hypersensitivity and neuronal hyperexcitability are mediated by rapid protein synthesis at the spinal level. Mol Pain 2009; 5: 27.

12. Zeng $\mathrm{H}$, Chi $\mathrm{H}$. mTOR signaling in the differentiation and function of regulatory and effector T cells. Curr Opin Immunol 2017; 46: 103-11.

13. Li JN, Sheets PL. The central amygdala to periaqueductal gray pathway comprises intrinsically distinct neurons differentially affected in a model of inflammatory pain. J Physiol 2018; 596: 6289-305.

14. Mills EP, Di Pietro F, Alshelh Z, Peck CC, Murray GM, Vickers $\mathrm{ER}$, et al. Brainstem pain-control circuitry connectivity in chronic neuropathic pain. J Neurosci 2018; 38: 465-73.

15. Ong WY, Stohler CS, Herr DR. Role of the prefrontal cortex in pain processing. Mol Neurobiol 2019; 56: 1137-66.

16. Shabab T, Khanabdali R, Moghadamtousi SZ, Kadir HA, Mohan G. Neuroinflammation pathways: a general review. Int J Neurosci 2017; 127: 624-33.

17. Gwak YS, Hulsebosch CE, Leem JW. Neuronal-glial interactions maintain chronic neuropathic pain after spinal cord injury. Neural Plast 2017; 2017: 2480689.

18. Yang Y, Zhang Z, Guan J, Liu J, Ma P, Gu K, et al. Administrations of thalidomide into the rostral ventromedial medulla alleviates painful diabetic neuropathy in Zucker diabetic fatty rats. Brain Res Bull 2016; 125: 144-51.

19. Goyal SN, Reddy NM, Patil KR, Nakhate KT, Ojha S, Patil CR, et al. Challenges and issues with streptozotocin-induced diabetes - a clinically relevant animal model to understand the diabetes pathogenesis and evaluate therapeutics. Chem Biol Interact 2016; 244: 49-63.

20. Swanson LW. Brain maps 4.0-structure of the rat brain: an open access atlas with global nervous system nomenclature ontology and flatmaps. J Comp Neurol 2018; 526: 935-43.

21. Um SW, Kim MJ, Leem JW, Bai SJ, Lee BH. Pain-relieving effects of mTOR inhibitor in the anterior cingulate cortex of neuropathic rats. Mol Neurobiol 2019; 56: 2482-94.

22. Fischer TZ, Waxman SG. Neuropathic pain in diabetes--evi- dence for a central mechanism. Nat Rev Neurol 2010; 6: 4626.

23. Bogush M, Heldt NA, Persidsky Y. Blood brain barrier injury in diabetes: unrecognized effects on brain and cognition. J Neuroimmune Pharmacol 2017; 12: 593-601.

24. Benarroch EE. Periaqueductal gray: an interface for behavioral control. Neurology 2012; 78: 210-7.

25. Tortorici V, Morgan MM. Comparison of morphine and kainic acid microinjections into identical PAG sites on the activity of RVM neurons. J Neurophysiol 2002; 88: 1707-15.

26. Guo J, Fu X, Cui X, Fan M. Contributions of purinergic P2X3 receptors within the midbrain periaqueductal gray to diabetes-induced neuropathic pain. J Physiol Sci 2015; 65: 99-104.

27. Zhuang X, Chen Y, Zhuang X, Chen T, Xing T, Wang W, et al. Contribution of pro-inflammatory cytokine signaling within midbrain periaqueductal gray to pain sensitivity in Parkinson's disease via GABAergic pathway. Front Neurol 2016; 7: 104.

28. Probert L. TNF and its receptors in the CNS: the essential, the desirable and the deleterious effects. Neuroscience 2015; 302: 2-22.

29. Loggia ML, Chonde DB, Akeju O, Arabasz G, Catana C, Edwards RR, et al. Evidence for brain glial activation in chronic pain patients. Brain 2015; 138(Pt 3): 604-15.

30. Tanaka T, Narazaki M, Kishimoto T. IL-6 in inflammation, immunity, and disease. Cold Spring Harb Perspect Biol 2014; 6: a016295.

31. Hoffman HM, Rosengren S, Boyle DL, Cho JY, Nayar J, Mueller JL, et al. Prevention of cold-associated acute inflammation in familial cold autoinflammatory syndrome by interleukin-1 receptor antagonist. Lancet 2004; 364: 1779-85.

32. Oka T, Aou S, Hori T. Intracerebroventricular injection of interleukin-1 beta enhances nociceptive neuronal responses of the trigeminal nucleus caudalis in rats. Brain Res 1994; 656: 236-44.

33. Hahm ET, Kim Y, Lee JJ, Cho YW. GABAergic synaptic response and its opioidergic modulation in periaqueductal gray neurons of rats with neuropathic pain. BMC Neurosci 2011; 12: 41.

34. Mahdian Dehkordi F, Kaboutari J, Zendehdel M, Javdani M. The antinociceptive effect of artemisinin on the inflammatory pain and role of GABAergic and opioidergic systems. Korean J Pain 2019; 32: 160-7. 